\title{
Circadian Regulation of Hepatic Cytochrome P450 2a5 by Peroxisome Proliferator-Activated Receptor $\gamma^{\mathbb{\S}}$
}

\author{
Jiangming Deng, Lianxia Guo, and Baojian Wu
}

Research Center for Biopharmaceutics and Pharmacokinetics, College of Pharmacy (J.D., L.G., B.W.) and Guangdong Province Key Laboratory of Pharmacodynamic Constituents of TCM and New Drugs Research (J.D., B.W.), Jinan University, Guangzhou, China

Received June 13, 2018; accepted August 17, 2018

\section{ABSTRACT}

Human CYP2A6 (Cyp2a5 in mice) plays an important role in metabolism and detoxification of various drugs and chemicals. Here, we investigated a potential role of peroxisome proliferatoractivated receptor $\gamma(\mathrm{Ppar}-\gamma$ ) in circadian regulation of the Cyp2a5 enzyme. We first showed that Cyp2a5 mRNA and protein in mouse liver displayed robust circadian oscillations. Consistent with a circadian protein pattern, Cyp2a5-mediated 7-hydroxylation of coumarin was circadian time-dependent. Formation of 7hydroxycoumarin was more extensive at a dosing time of Zeitgeber time 2 (ZT2) than that at ZT14. Interestingly, the nuclear receptor Ppar- $\gamma$ was also a circadian gene. Circadian Ppar- $\gamma$ protein level was strongly correlated with the Cyp2a5 mRNA level $(r=0.989)$.
Furthermore, Ppar- $\gamma$ activation (by a selective agonist, rosiglitazone) upregulated Cyp2a5 expression in Hepa-1c1c7 cells, whereas Ppar- $\gamma$ knockdown downregulated Cyp2a5 expression. Also, Ppar- $\gamma$ knockdown blunted the rhythmicity of Cyp2a5 mRNA in serum-shocked Hepa-1c1c7 cells. In addition, a combination of promoter truncation analysis, mobility shift, and chromatin immunoprecipitation assays revealed that Ppar- $\gamma$ directly bound to a PPAR response element (i.e., the -1418 - to $-1396-b p$ region) within Cyp2a5 promoter and activated the gene transcription. Taken together, Ppar- $\gamma$ was a transcriptional activator of Cyp2a5, and its rhythmic expression contributed to circadian expression of Cyp2a5.
Introduction

Cytochrome P450s (P450s) are a large superfamily of enzymes that catalyze the oxidation and reduction of chemicals. P450s are the principal enzymes for drug metabolism and detoxification, accounting for approximately $75 \%$ of all drug metabolism (Guengerich, 2008). In addition, P450s play important roles in many biologic processes, including hormone synthesis (steroidogenesis), cholesterol synthesis, and vitamin D metabolism (Hanukoglu, 1992; Lorbek et al., 2012; Jones et al., 2014). Human P450s contain a total of 57 members that are divided into 18 families (Nelson et al., 2004). The main contributors to xenobiotic metabolism are usually from families 1 to 3 (Guengerich, 2008). Similar to humans, mice have 18 families of $P 450$ enzymes. However, mouse $\mathrm{P} 450$ s possess a greater number of members $(n=105)$ (Hrycay and Bandiera, 2009). The mouse model is regarded as the most similar to humans with respect to catalytic activities of certain P450 enzymes (e.g., CYP3A4) (Bogaards et al., 2000; Muruganandan and Sinal, 2008). In fact, about 40 orthologous pairs of mouse-human P450 genes have been identified that perform similar or identical functions (Guengerich, 1997; Nelson et al., 2004; Choudhary et al., 2005).

This work was supported by the National Natural Science Foundation of China [Grants 81722049 and 81573488].

https://doi.org/10.1124/dmd.118.083071.

S This article has supplemental material available at dmd.aspetjournals.org.
Human CYP2A6, highly expressed in the liver (Shimada et al., 1994), plays an important role in metabolism and detoxification of various drugs (e.g., valproic acid, disulfiram, and losigamone) and chemicals (e.g., coumarin and nicotine) (Malaiyandi et al., 2005). In addition, CYP2A6 bioactivates several procarcinogens (e.g., aflatoxin B1, $N$ nitrosonornicotine, and nicotine-derived nitrosamine ketone) to mutagenic products (Patten et al., 1997; Fujita and Kamataki, 2001). Mouse Cyp2a5 is an ortholog of human CYP2A6 (Honkakoski and Negishi, 1997). Both CYP2A6 and Cyp2a5 are well characterized coumarin 7-hydroxylases. 7-Hydroxylation is the predominant metabolic pathway for coumarin in humans (Miles et al., 1990). However, it is a relatively minor route for coumarin clearance in mice (and rats) because, in rodents, coumarin is preferentially metabolized to coumarin 3,4epoxide, which rearranges to o-hydroxyphenylacetaldehyde (Lake, 1999).

The efficacy and toxicity of many drugs are dependent on the time of administration (Dallmann et al., 2014, 2016). For instance, the anticancer effects of 5-fluorouracil show a dosing-time dependency (Lévi et al., 2010). Hepatotoxicity of acetaminophen is more severe during the night than the daytime (DeBruyne et al., 2014). It is believed that time-dependent drug activity and toxicity are regulated by the circadian clock (Dallmann et al., 2014, 2016). The clock system underlies the circadian rhythms and is essentially a transcriptionaltranslational feedback loop system mainly consisting of CLOCK, BMAL1, CRY, and PER proteins (Partch et al., 2014). CLOCK and BMAL1 form a heterodimer that activates the transcription of

ABBREVIATIONS: ANOVA, analysis of variance; ChIP, chromatin immunoprecipitation; DBP, albumin D-site-binding protein; EMSA, electrophoretic mobility shift assay; P450, cytochrome P450; PPAR- $\gamma$, peroxisome proliferator-activated receptor $\gamma$; PPRE, PPAR response element; qPCR, quantitative polymerase chain reaction; siPpar- $\gamma$, siRNA targeting Ppar- $\gamma$; siRNA, short interfering RNA; UPLC-QTOF/MS, ultraperformance liquid chromatography/quadrupole time-of-flight mass spectrometry; ZT, Zeitgeber time. 
clock-controlled genes, including $C R Y$ and PER (Reppert and Weaver, 2002). Once reaching a critical level, CRY and PER proteins in turn inhibit the activities of BMAL1/CLOCK, thereby generating circadian oscillations of clock-controlled genes (Reppert and Weaver, 2002). Interestingly, the circadian clock is able to regulate drug-processing genes (e.g., Cyp2b10 and $M d r$ ), possibly contributing to the time dependency of drug activity/toxicity (Gachon et al., 2006; Murakami et al., 2008). Cyp2a5 has been identified as a circadian gene, and the albumin D-site-binding protein (DBP) is a transcriptional factor potentially controlling its circadian expression (Lavery et al., 1999). However, the mild fluctuation after DBP deletion suggests additional factors accounting for circadian expression of Cyp2a5 (Lavery et al., 1999).

Peroxisome proliferator-activated receptor $\gamma(\operatorname{PPAR}-\gamma)$ belongs to the PPAR subfamily of nuclear receptors (the other two members of PPAR subfamily are PPAR- $\alpha$ and PPAR- $\delta$ ). PPAR proteins regulate gene transcription by dimerizing with retinoid $X$ receptor and binding to the PPAR response element (PPRE) (Desvergne and Wahli, 1999). PPAR- $\alpha$ and PPAR- $\gamma$ mainly function to regulate fatty acid storage and glucose metabolism (Jones et al., 2005). Their agonists (e.g., gemfibrozil, fenofibrate, troglitazone, and rosiglitazone) have been used in the treatment of hyperlipidemia and hyperglycemia ( $\mathrm{Li}$ et al., 2008). Extensive studies have revealed PPAR- $\alpha$ as an important regulator of drug biotransformation (including phase I and phase II biotransformation reactions) (Rakhshandehroo et al., 2010; Thomas et al., 2013). Interestingly, PPAR- $\gamma$ has been reported to regulate UGT1A9, a phase II enzyme (Barbier et al., 2003). A recent study also show the regulation of fatty acid-metabolizing protein CYP4V2 by PPAR- $\gamma$ (Yi et al., 2017). However, whether and how PPAR- $\gamma$ regulates phase I drug metabolism remain unknown.

In this study, we aimed to investigate a potential role of Ppar- $\gamma$ in circadian regulation of Cyp2a5. We first determined the circadian rhythmicity of both Cyp2a5 and Ppar- $\gamma$ in mouse liver and analyzed the time-dependent activities of Cyp2a5 using coumarin (7-hydroxylation) as a functional marker. The regulatory effects of Ppar- $\gamma$ on Cyp2a5 were assessed in mouse hepatoma Hepa-1c1c7 cells. Regulation of Cyp2a5 rhythmicity by Ppar- $\gamma$ was confirmed using serum-shocked cells. Transcriptional regulation of Cyp2a5 by Ppar- $\gamma$ was investigated using a combination of promoter analysis, mobility shift, and chromatin immunoprecipitation (ChIP) assays. Our study is the first to demonstrate that Ppar- $\gamma$ participates in circadian regulation of Cyp2a5.

\section{Materials and Methods}

Materials. Coumarin and 7-hydroxycoumarin were purchased from Aladdin (Shanghai, China). Rosiglitazone was purchased from Sigma-Aldrich (St. Louis, MO).
Anti-Cyp2a5 antibody (catalog number ab3570) was purchased from Abcam (Cambridge, MA). Anti-Ppar- $\gamma$ antibody (catalog number sc-7273) was obtained from Santa Cruz Biotechnology (Santa Cruz, CA). Anti-IgG antibody was purchased from Cell Signaling Technology (Danvers, MA). Short interfering RNA (siRNA) targeting Ppar- $\gamma$ (siPpar- $\gamma$ ) was obtained from TranSheep Bio-Tech (Shanghai, China).

Animal Studies. All animal care and experimental procedures were in compliance with guidelines approved by the Institute of Laboratory Animal Science of Jinan University (Guangzhou, China). All C57BL/6 mice, receiving food and water ad libitum, were housed in a temperature- and humidity-controlled room with a 12-hour light/dark cycle (light on at 7 a.m. and off at 7 p.m.). For the first set of studies, six mice ( 8 weeks old, male) were sacrificed at each circadian time point [i.e., Zeitgeber time 2 (ZT2), ZT6, ZT10, ZT14, ZT18, and ZT22], and the livers were isolated, snap-frozen, and stored at $-80^{\circ} \mathrm{C}$ until processing for mRNA and protein analyses.

For the second set of studies, mice ( 8 weeks old, male) were randomly divided into two groups ( $n=30$ per group). One group of mice were injected (i.p.) with $30 \mathrm{mg} / \mathrm{kg}$ coumarin at the circadian time ZT2. The other group of mice were injected (i.p.) with $30 \mathrm{mg} / \mathrm{kg}$ coumarin at ZT14. At each time point $(5,10,15,20$, 30 , and 45 minutes), five mice were sacrificed to collect plasma samples. Drug quantification of coumarin and its 7-hydroxylated metabolite was performed using the ultra-performance liquid chromatography/quadrupole time-of-flight mass spectrometry (UPLC-QTOF/MS) system (Waters, Milford, MA). Pharmacokinetic data were analyzed using the noncompartmental approach with WinNonlin software version 7.0 (Pharsight, Mountain View, CA).

Plasmid Construction. Mouse Ppar- $\gamma$ gene (GenBank accession number: NM_011146.3) was synthesized and cloned into the expression vector $\mathrm{p} 3 \mathrm{xFLAG-}$ CMV-10. Various versions of Cyp2a5 promoter luciferase constructs $(-2000 /$ $+25,-1500 /+60,-800 /+25$, and $-50 /+25$ ) were synthesized and cloned into the pGL4.10 vector. After verification by DNA sequencing, the plasmids were transformed into Escherichia coli JM109 and purified using EasyPure HiPure Plasmid MiniPrep kits (TransGen Biotech, Beijing, China).

Cell Culture. Hepa-1c1c7 cells were purchased from American Type Culture Collection (Manassas, VA) and were maintained in Dulbecco's modified Eagle's medium supplemented with $10 \%$ fetal bovine serum at $37^{\circ} \mathrm{C}$ in a humidified $5 \%$ $\mathrm{CO}_{2}$ atmosphere.

Serum-Shock Experiment. To stimulate circadian gene expression in cultured Hepa-1c1c7 cells, a serum-shock experiment was performed as previously described (Matsunaga et al., 2012). In brief, the cells were grown in Dulbecco's modified Eagle's medium supplemented with $10 \%$ fetal bovine serum and treated with siPPAR- $\gamma$ (or siNC [siNC, short interfering RNA for negative control] as the control) for 24 hours. This was followed by incubation in serum-starved medium for 12 hours. On the day of serum shock, $50 \%$ fetal bovine serum was added for 2 hours, and then the cells were changed back to starvation medium. The cells were collected for RNA extraction at 0, 2, 4, 6, and 8 hours after the serum shock.

Reverse-Transcription Quantitative Polymerase Chain Reaction. Reverse-transcription quantitative polymerase chain reaction (qPCR) was performed as previously described (Zhao et al., 2018). In brief, total RNA was extracted using RNAiso Plus reagent (Takara Bio, Shiga, Japan) and used as a template for cDNA synthesis (reverse transcription). The PCR system consisted of

TABLE 1

Oligonucleotides used in this study

\begin{tabular}{lcr}
\hline & Forward $\left(5^{\prime}-3^{\prime}\right)$ & Reverse $\left(5^{\prime}-3^{\prime}\right)$ \\
\hline RT-qPCR & & \\
Cyp2a5 & GGACAAAGAGTTCCTGTCACTGCTTC & GTGTTCCACTTTCTTGGTTATGAAGTCC \\
mPPIB & TCCACACCCTTTCCGGTCC & CAAAAGGAAGACGACGGAGC \\
mPpar- $\gamma$ & AACCCACAACCAAATCCACAC & ATCACGGAGAGGTCCACAGA \\
mRxr $\alpha$ & ATGGACACCAAACATTTCCTGC & CTCGACCCGTTGGAGAGTT \\
EMSA & & TTTAGACAGGTGGAGATGGGTGAGGTTGG \\
Cyp2a5 & CCAACCTCACCCATCTCCACCTGTCTAAA & TTTAGGAAGGTGCAGACAGGCTAGGTTGG \\
Cyp2a5 (mutant) & CCAATCTCAACCAGATCCCGCTTTCTAAA & \\
ChIP & & \\
Cyp2a5 & TGAGGCTGGTGATGTGCCA & GACCCTACTGTTGCCCCATA \\
sema3g & GCTGGGTGTCTGGCTGAG & GACTGCTTCTGTCTCTGTTTCC \\
\hline
\end{tabular}

mPpar- $\gamma$, mouse peroxisome proliferator-activated receptor gamma; mPPIB, mouse peptidylprolyl isomerase B; RT, reverse-transcription; mRxr $\alpha$, mouse retinoid X receptor alpha. 

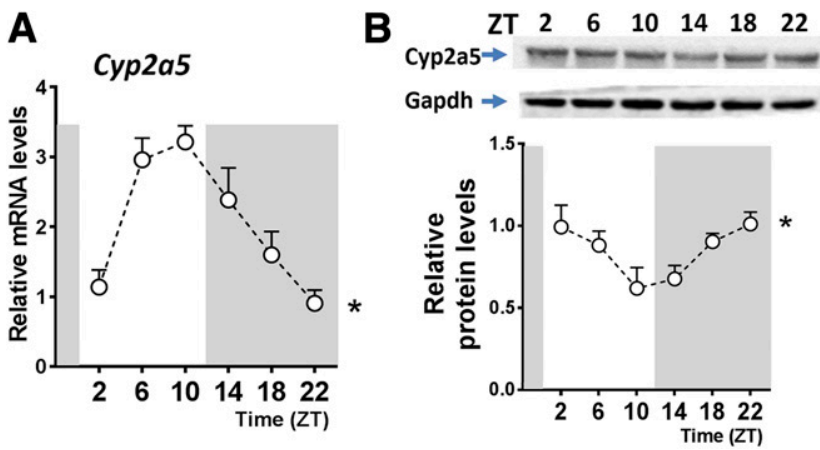

Fig. 1. Circadian (24-hour) rhythms of Cyp2a5 mRNA (A) and protein (B) in mouse liver. Data are the mean \pm S.D. $(n=6) . * P<0.05$ (one-way ANOVA). Oneway ANOVA was used to analyze the significance of the 24-hour variation in mRNA and protein expression. Gapdh, glyceraldehyde-3-phosphate dehydrogenase.

$50 \mathrm{ng}$ of cDNA, $0.2 \mu \mathrm{M}$ PCR primers, and GoTaq qPCR Master Mix (Promega, Madison, WI). The cycling conditions were initial denaturation at $95^{\circ} \mathrm{C}$ for 5 minutes followed by 40 cycles of denaturation at $95^{\circ} \mathrm{C}$ for 15 seconds, annealing at $60^{\circ} \mathrm{C}$ for 30 seconds, and extension at $72^{\circ} \mathrm{C}$ for 30 seconds. Peptidylprolyl isomerase $\mathrm{B}$ was used as an internal control. The relative expression was determined using the $2^{-\Delta \Delta C T}$ method. All primer sequences are summarized in Table 1.

Luciferase Reporter Assay. Hepa-1c1c7 cells were seeded in a 48-well plate at a density of $5 \times 10^{4}$ cells/well. Once reaching semiconfluence, the cells were transfected with $500 \mathrm{ng}$ of Cyp2a5 luciferase (firefly) reporter plasmid, $50 \mathrm{ng}$ of pRL-TK vector, and $200 \mathrm{ng}$ of Ppar- $\gamma$ (or blank plasmid). The next day, the cells were treated with rosiglitazone (a selective PPAR- $\gamma$ agonist) or vehicle. After 24-hour treatment, cells were lysed in $45 \mu$ l of passive lysis buffer. The cell lysate was collected to determine the luciferase activities using the Dual-Luciferase Reporter Assay System and GloMax 20/20 luminometer (Promega). Firefly luciferase activities were normalized to renilla luciferase values and expressed as relative luciferase units.

Western Blotting. Protein samples were prepared in RIPA (radioimmunoprecipitation assay) lysis buffer (Beyotime, Shanghai, China) containing $1 \mathrm{mM}$ PMSF (phenylmethylsulfonyl fluoride). Protein concentrations were determined by the bicinchoninic acid assay (Beyotime). Forty-microgram samples were loaded to SDS-polyacrylamide gel electrophoresis (10\% acrylamide gels) and transferred onto a PVDF (polyvinylidene difluoride) membrane (Millipore, Bedford, MA). After blocking with 5\% nonfat milk in Tris-buffered saline/Tween 20 , the membrane was reacted with primary antibody (1:500 dilution for antiCyp2a5 and 1:200 dilution for anti-Ppar- $\gamma$ ), followed by reacting with horseradish peroxidase-conjugated secondary antibody (1:5000 dilution). Glyceraldehyde-3-phosphate dehydrogenase was used as a loading control. The protein bands were visualized using the Omega Lum G imaging system (Aplegen, Pleasanton, CA) after adding enhanced chemiluminescence.

Electrophoretic Mobility Shift Assay. Nuclear proteins from Hepa-1c1c7 cells were isolated using a cytoplasmic/nuclear protein extraction kit (Beyotime). Biotin-labeled probes were prepared by annealing complementary sense and antisense oligonucleotides (Table 1). Electrophoretic mobility shift assays (EMSAs) were performed using a chemiluminescent EMSA kit (Beyotime). In brief, $6 \mu \mathrm{g}$ of nuclear protein was mixed well in EMSA binding buffer and incubated at room temperature for 10 minutes. Then, the probe $(200 \mathrm{fmol})$ was added to the mixture and incubated for another 20 minutes. For competition reactions, a 50-fold molar excess of unlabeled wide-type or mutated probes was added to incubate with nuclear proteins prior to reacting with biotin-labeled probe. The DNA - protein complexes were subjected to $4 \%$ nondenaturing polyacrylamide gel electrophoresis and transferred onto a Hybond-N+ membrane (Amersham, Buckinghamshire, UK). The membrane was exposed to UV rays for 10 minutes. After blocking, washing, and balancing, the membrane was treated with enhanced chemiluminescence reagent and visualized by the Omega Lum G imaging system (Aplegen).

ChIP. ChIP assays were performed using the Enzymatic Chromatin IP Kit (Cell Signaling Technology, Beverly, MA) according to the manufacturer's instructions. In brief, the fresh liver tissue of mouse was cross-linked with $1.5 \%$
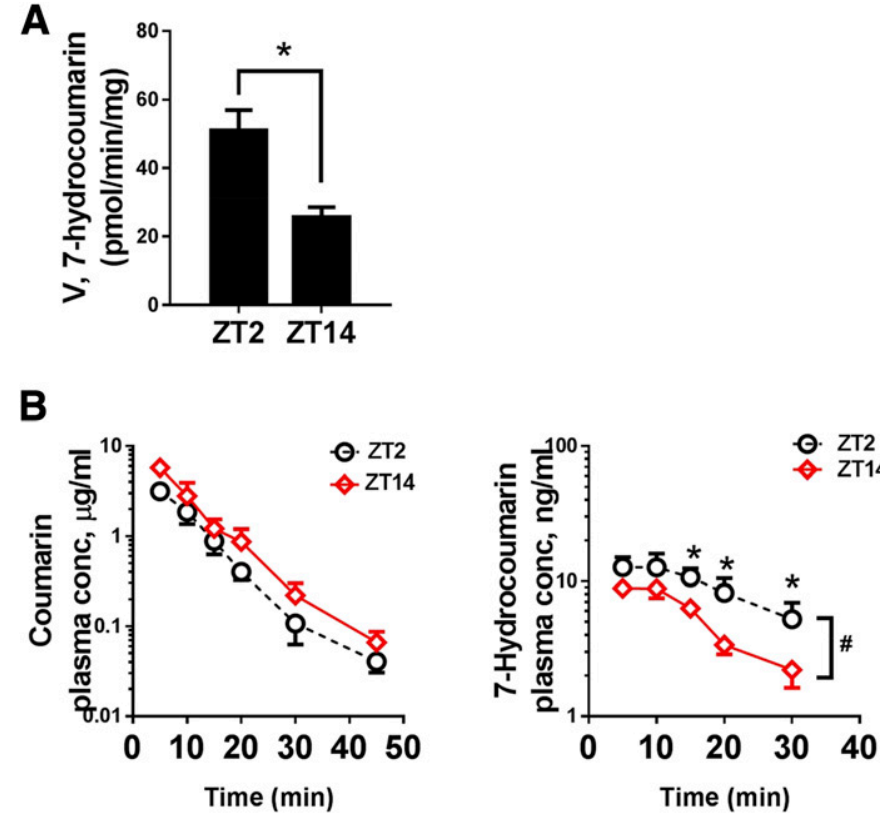

Fig. 2. Time-dependent metabolism of coumarin in vitro and in vivo. (A) Liver microsomal metabolism assays of coumarin. Microsomes were prepared using mouse livers collected at ZT2 and ZT14, respectively. Data are the mean \pm S.D. $(n=6)$. $* P<0.05$ ( $t$ test). (B) Plasma concentration-time profiles for coumarin and 7-hydroxycoumarin in mice after coumarin administration $(30 \mathrm{mg} / \mathrm{kg}$, i.p., $n=5)$ at ZT2 or ZT14. "Significant effect of dosing time on drug concentrations $(P<0.05$, two-way ANOVA); $* P<0.05$ compared with the value for the ZT14 group at the corresponding times (two-way ANOVA followed by Tukey's post hoc test).

formaldehyde for 20 minutes at room temperature. Then, glycine was used to stop the cross-linking. The chromatin was digested with micrococcal nuclease and sheared into small fragments by sonication. After immunoprecipitation with antiPpar- $\gamma$, chromatin was de-cross-linked at $65^{\circ} \mathrm{C}$ for 2 hours and purified using spin columns. At last, the samples were analyzed by quantitative real-time PCR with the purified DNA as a template.

Liver Microsomal Metabolism Assay. The livers were collected from the mice sacrificed at ZT2 and ZT14. Liver microsomes were prepared by sequential ultracentrifugation, first at $9000 \mathrm{~g}$ for 10 minutes and then at $100,000 \mathrm{~g}$ for 60 minutes (Zhang et al., 2018). The incubation mixture (a total volume of $200 \mu \mathrm{l}$ ) contained $0.3 \mathrm{mg} / \mathrm{ml}$ liver microsomes, $1.55 \mathrm{mM}$ NADPH, and $1 \mu \mathrm{M}$ coumarin. The incubation reaction was performed at $37^{\circ} \mathrm{C}$ for 2 hours (under a linear condition) and was terminated by adding $200 \mu \mathrm{l}$ acetonitrile. The resultant mixture was centrifuged at $15,000 \mathrm{~g}\left(4^{\circ} \mathrm{C}\right)$ for 15 minutes, and the supernatant was subjected to UPLC-QTOF/MS analysis.

Quantification of Coumarin and 7-Hydroxycoumarin. Coumarin and 7-hydroxycoumarin were quantified using a UPLC-QTOF/MS system (Waters) and a BEH C18 column $(2.1 \times 50 \mathrm{~mm}, 2.6 \mu \mathrm{m}$; Waters $)$. The mobile phase was $0.1 \%$ formic acid (mobile phase A) and $0.1 \%$ formic acid in acetonitrile (mobile phase $\mathrm{B}$ ). The flow rate was set at $0.3 \mathrm{ml} / \mathrm{min}$. The gradient elution program was $5 \% \mathrm{~B}$ at $0-1$ minute, $5 \%-95 \% \mathrm{~B}$ at $1-3$ minutes, $95 \% \mathrm{~B}$ at $3-3.5$ minutes, and $95 \%-10 \%$ B at $3.5-5$ minutes. A mass spectrometer was operated at the positive ion full-scan mode as previously described (Zhao et al., 2018). Peak areas of coumarin and 7-hydroxycoumarin were recorded with extract masses of $\mathrm{m} / \mathrm{z}$ 147.041 and 163.038 Da, respectively. The calibration curves for coumarin and 7-hydroxycoumarin were linear $\left(r^{2}>0.99\right)$ over the entire concentration range (i.e., $15.6-10,000 \mathrm{ng} / \mathrm{ml}$ for coumarin and $1.56-100 \mathrm{ng} / \mathrm{ml}$ for 7-hydroxycoumarin). The limits of detection (defined as a signal:noise ratio of $3: 1$ ) were 1 and $0.1 \mathrm{ng} / \mathrm{ml}$ for coumarin and 7-hydroxycoumarin, respectively. The limits of quantitation (defined as a signal:noise ratio of 10:1) were 10 and $1 \mathrm{ng} / \mathrm{ml}$ for coumarin and 7-hydroxycoumarin, respectively.

Statistical Analysis. Data are presented as the mean \pm S.D. The significance of the 24-hour variation in mRNA or protein expression was tested by one-way analysis of variance (ANOVA). Student's $t$ test was used to test for statistical differences between two groups, whereas ANOVA followed by Tukey's post hoc 
TABLE 2

Pharmacokinetic parameters for mice after administration of coumarin (i.p.)

\begin{tabular}{lcccc}
\hline Group & $\mathrm{t}_{1 / 2}$ & $\mathrm{~T}_{\max }$ & $\mathrm{C}_{\max }$ & $\mathrm{AUC}_{0-\mathrm{t}}$ \\
\hline & $\min$ & $\min$ & $\mu g / m l$ & $(\mu g / m l) * \min$ \\
Coumarin & & & & \\
ZT2 & $7.44 \pm 0.98$ & 5 & $3.15 \pm 0.17$ & $48.14 \pm 6.15$ \\
ZT14 & $6.44 \pm 0.52$ & 5 & $4.19 \pm 0.92$ & $56.34 \pm 7.53$ \\
7-Hydroxycoumarin & & & & \\
ZT2 & - & 11.67 & $0.0127 \pm 0.0023$ & $0.33 \pm 0.01$ \\
ZT14 & - & 6.67 & $0.0093 \pm 0.0006$ & $0.18 \pm 0.01 *$
\end{tabular}

$\mathrm{AUC}_{0-\mathrm{t}}$, area under the plasma concentration-time curve.

$* P<0.05$ vs. ZT2 group.

test was used for multiple group comparison. For serum-shock experiments, the data were analyzed by a two-way ANOVA followed by Tukey's post hoc test (the mRNA expression as the outcome variable, siRNA treatment and sampling time post treatment as two independent factors; the interaction effect was included in the analysis). Pharmacokinetic data were also analyzed by two-way ANOVA followed by Tukey's post hoc test (the drug concentration as the outcome variable, dosing time and sampling time post dosing as two independent factors; the interaction effect was included in the analysis). The homogeneity of variance assumption for ANOVAs was verified by Levene's test. All statistical analyses were performed using GraphPad Prism 7.0 (GraphPad Software, San Diego, CA). A level of probability of $5 \%$ was considered significant.

\section{Results}

Circadian Expression and Activity of Cyp2a5 in Mouse Liver. The Cyp2a5 mRNA in mouse liver displayed a robust circadian oscillation with a zenith at ZT10 (Fig. 1A). Interestingly, hepatic Cyp2a5 protein showed a circadian rhythm with a nadir at ZT10 (Fig. 1B). The circadian pattern of mRNA was in antiphase to that of protein, suggesting a delay of about 12 hours in the translation of mRNA to protein for Cyp2a5. Coumarin (7-hydroxylation), a known functional marker for Cyp2a5, was used to probe the circadian activity of Cyp2a5. Based on in vitro microsomal metabolism assay, hepatic Cyp2a5 showed a higher activity toward 7-hydroxylation of coumarin at ZT2 than at ZT14, consistent with a higher Cyp2a5 expression at ZT2 (Fig. 2A). Pharmacokinetic studies were further performed to compare the metabolism and pharmacokinetics of coumarin after drug dosing at two different circadian time points (i.e., ZT2 and ZT14). The dosing time had a minor effect on the plasma coumarin concentration-time profile (Fig. 2B). The area under the curve value (i.e., representing the systemic exposure) was slightly smaller (but not statistically different) at ZT2 than at ZT14 (Table 2). By contrast, compared with ZT14, ZT2 dosing showed higher plasma 7-hydroxycoumarin concentrations (Fig. 2B). Accordingly, the area under the curve value for 7-hydroxycoumarin was much larger at ZT2 than ZT14 (Table 2). Taken together, these data indicated that the expression and activity of hepatic Cyp2a5 were circadian time-dependent.

Circadian Expression of Ppar- $\boldsymbol{\gamma}$ in Mouse Liver. Ppar- $\gamma$ mRNA in mouse liver also showed a circadian fluctuation with a peak at ZT6 (Fig. 3A). Likewise, circadian oscillation was observed for hepatic Ppar$\gamma$ protein, and the protein level peaked at ZT10 (Fig. 3B). Interestingly, the Ppar- $\gamma$ protein level was strongly correlated with the Cyp2a5 mRNA level in the liver $\left(r^{2}=0.98\right.$; Fig. 3C). This suggested a potential role of Ppar- $\gamma$ in circadian regulation of Cyp2a5.

Ppar- $\gamma$ Positively Regulates Cyp2a5 in Hepa-1C1c7 Cells. We next investigated the regulatory effects of Ppar- $\gamma$ on Cyp2a5 using mouse hepatoma Hepa-1c1c7 cells. After treatment of the cells with rosiglitazone (a selective Ppar- $\gamma$ agonist; Edvardsson et al., 1999), both Cyp2a5 mRNA (Fig. 4A) and protein (Fig. 4B) were significantly

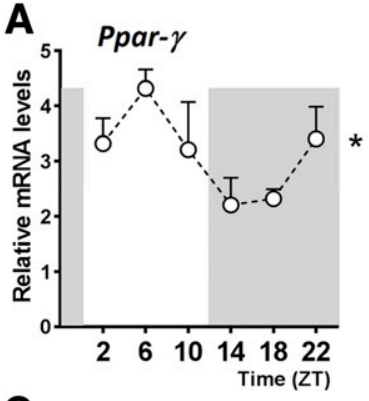

B $\begin{array}{lllllll}\text { ZT } & 2 & 6 & 10 & 14 & 18 & 22\end{array}$

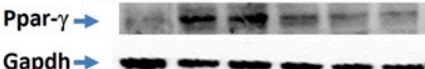
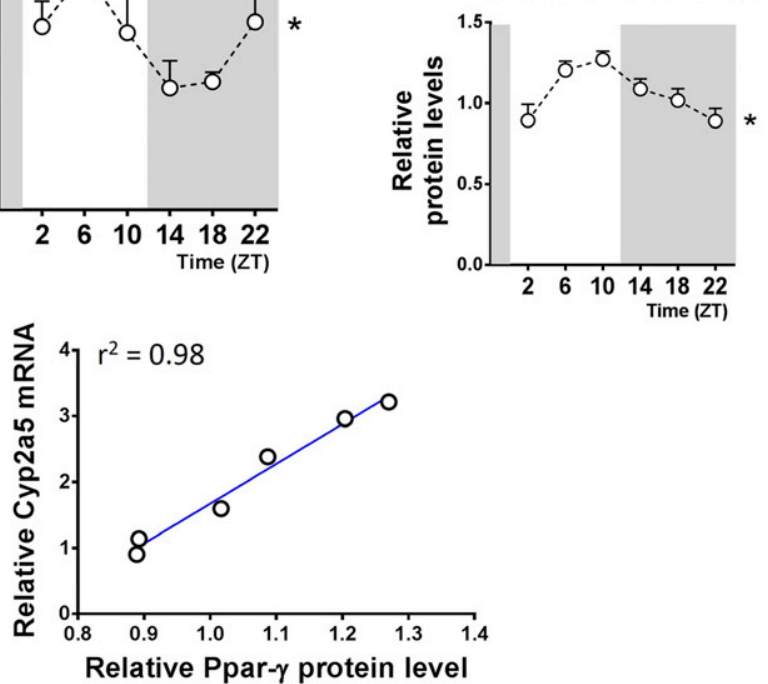

Fig. 3. Circadian (24-hour) rhythms of Ppar- $\gamma$ mRNA (A) and protein (B) in mouse liver. Data are the mean \pm S.D. $(n=6) * *<0.05$ (one-way ANOVA). One-way ANOVA was used to analyze the significance of the 24-hour variation in mRNA and protein expression. (C) Correlation analysis of Ppar- $\gamma$ protein with Cyp2a5 mRNA levels. Gapdh, glyceraldehyde-3-phosphate dehydrogenase.

increased. Similarly, Ppar- $\gamma$ regulated human CYP2A6 in HepG2 cells (Supplemental Fig. 1). We also tried to assess the effects of Ppar- $\gamma$ knockdown on Cyp2a5 expression. Knockdown of Ppar- $\gamma$ was successfully achieved using a specific siRNA (termed siPpar- $\gamma$ ) (Fig. 5, A and B). Ppar- $\gamma$ knockdown resulted in reduced levels of both Cyp2a5 mRNA and protein (Fig. 5, A and B). Furthermore, rosiglitazone regulated Cyp2a5 in a Ppar- $\gamma$-dependent manner (Fig. 5C). Taken together, these data indicated that Ppar- $\gamma$ functioned as a positive regulator of Cyp2a5.
A

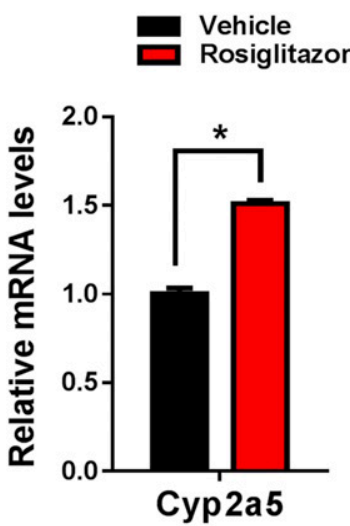

B

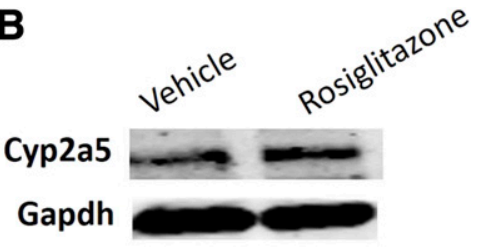

Gapdh

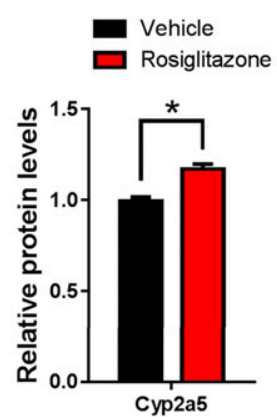

Fig. 4. Effects of Ppar- $\gamma$ activation on Cyp2a5 mRNA (A) and protein (B) in Hepa$1 \mathrm{c} 1 \mathrm{c} 7$ cells. Hepa-1c1c7 cells were seeded onto six-well plates. Twenty-four hours later, the cells were treated with rosiglitazone $(10 \mu \mathrm{M})$ for 24 hours. Ppar- $\gamma$ and Cyp2a5 mRNAs were quantified by reverse-transcription qPCR. Ppar- $\gamma$ and Cyp2a5 proteins were quantified by Western blotting. Data shown are the mean \pm S.D. $(n=$ 4). ${ }^{*} P<0.05$ ( $t$ test). Gapdh, glyceraldehyde-3-phosphate dehydrogenase. 
A

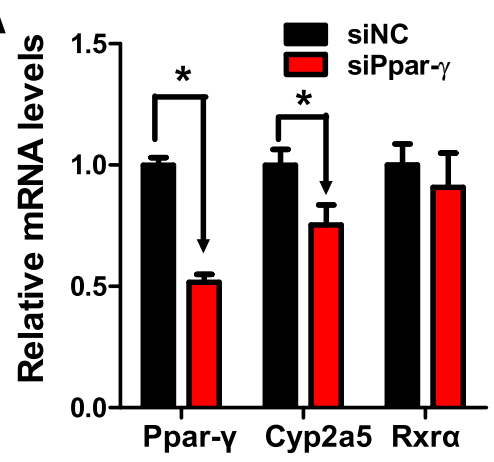

C

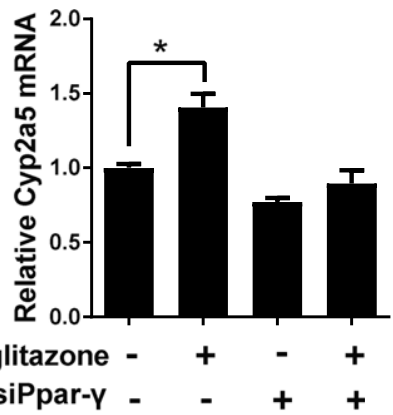

Fig. 5. Effects of Ppar- $\gamma$ knockdown on Cyp2a5 expression in Hepa-1c1c7 cells. (A) Effects of Ppar- $\gamma$ knockdown on Cyp2a5 mRNA. (B) Effects of Ppar- $\gamma$ knockdown on Cyp2a5 protein. (C) Ppar- $\gamma$-dependent regulation of Cyp2a5 by rosiglitazone. Hepa$1 \mathrm{c} 1 \mathrm{c} 7$ cells were seeded into six-well plates and transfected with $50 \mathrm{nM}$ siPpar- $\gamma$ or siNC (negative control) for 24 hours. In (C), siPpar- $\gamma$-transfected cells were treated with rosiglitazone $(10 \mu \mathrm{M})$ or vehicle for another 24 hours. Ppar- $\gamma$ and Cyp2a5 mRNAs were quantified by reverse-transcription qPCR. Ppar- $\gamma$ and Cyp2a5 proteins were quantified by Western blotting. Data shown are the mean \pm S.D. $(n=4)$. ${ }^{*} P<0.05$ ( $t$ test $)$. Gapdh, glyceraldehyde-3phosphate dehydrogenase; $\operatorname{Rxr} \alpha$, retinoid X receptor $\alpha$ siNC; short interfering RNA for negative control.
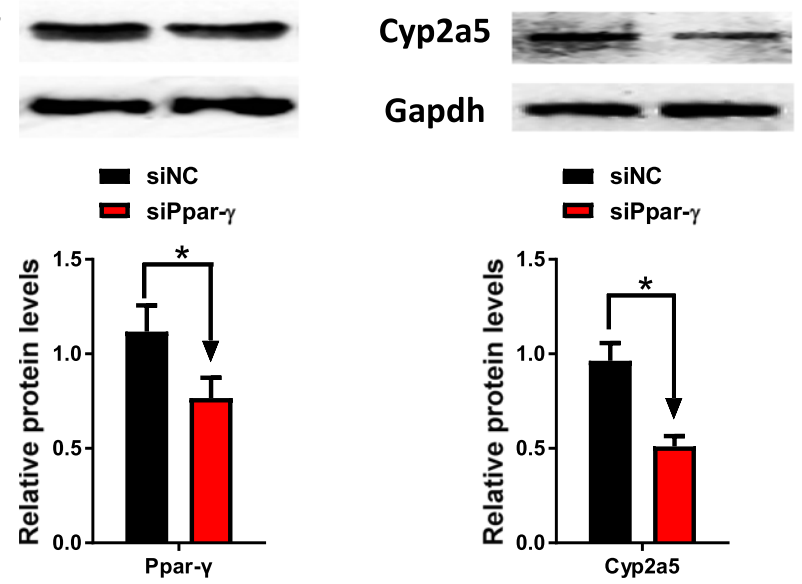

Ppar- $\gamma$ Knockdown Blunts Cyp2a5 Rhythmicity in SerumShocked Hepa-1c1c7 Cells. We further explored whether the Ppar- $\gamma$ receptor regulated the circadian rhythm of Cyp2a5. Time-dependent expression of Cyp2a5 in the cells was generated using the serum-shock method (Balsalobre et al., 1998). A serum shock induced circadian gene expression in Hepa-1c1c7 cells (e.g., Ppar- $\gamma$ and Cyp2a5 in Fig. 6). Treatment of the cells with siPpar- $\gamma$ resulted in reduced Ppar- $\gamma$ expression, as expected (Fig. 6A). In the meantime, siPpar- $\gamma$ blunted the rhythmicity of Cyp2a5 mRNA (Fig. 6B). The data suggested a critical role of Ppar- $\gamma$ in circadian regulation of Cyp2a5.

Ppar- $\boldsymbol{\gamma}$ Is a Transcriptional Activator of Cyp2a5. A Cyp2a5 luciferase reporter (with $-2.0-\mathrm{kb}$ proximal promoter) was constructed to investigate the potential role of Ppar- $\gamma$ in transcriptional regulation of Cyp2a5. The Ppar- $\gamma$ agonist rosiglitazone dose-dependently enhanced the promoter activity of Cyp2a5, suggesting a positive role of Ppar- $\gamma$ in transcriptional regulation of Cyp2a5 (Fig. 7A). The computational algorithm Genomatix (Genomatix AG, Munich, Germany) predicted four potential Ppar- $\gamma$ binding sites (i.e., A-, B-, C-, and D-sites) in Cyp2a5 promoter (Fig. 7B). Accordingly, three shorter (truncated) promoter constructs (i.e., $-1.5-,-0.8-$, and $-0.05-\mathrm{kb}$ constructs) were prepared for pinpointing the exact site for Ppar- $\gamma$ binding (Fig. 7B). Cotransfection with Ppar- $\gamma$ expression vector led to 95- and 55-fold increases in transcriptional activities for the 2.0 - and $1.5-\mathrm{kb}$ constructs, respectively (Fig. 7B). By contrast, the activities of $0.8-$ and $0.05-\mathrm{kb}$ constructs were unaffected in the presence of Ppar- $\gamma$ vector (Fig. 7B). The data indicated that the Ppar- $\gamma$ binding site was located between -1.5 and $-0.8 \mathrm{~kb}$ (probably the B-site).

EMSA assay was performed to determine whether Ppar- $\gamma$ binds to the B-site (i.e., a PPRE) in the Cyp2a5 promoter. Interestingly, the B-site was able to form a DNA-protein complex in the presence of nuclear proteins (Fig. 8A). The complex band disappeared in the presence of unlabeled probe but reappeared in the presence of mutated unlabeled probe (Fig. 8A). The results suggested that Ppar- $\gamma$ protein can bind to the $\mathrm{B}$-site of Cyp2a5 promoter. ChIP assay was also performed to explore the interactions of Cyp2a5 promoter with Ppar- $\gamma$ protein in vivo. Binding ability was assessed by enrichment of qPCR signals in the antiPpar- $\gamma$ sample in relation to the anti-IgG sample. The Cyp2a5-PPRE enrichment for the anti-Ppar- $\gamma$ sample was markedly higher than that for the anti-IgG sample, indicating significant recruitment of Ppar- $\gamma$ protein to this PPRE (B-site) (Fig. 8B). A similar result was shown for Sema3g (a positive oligonucleotide for Ppar- $\gamma$ binding) (Liu et al., 2015)
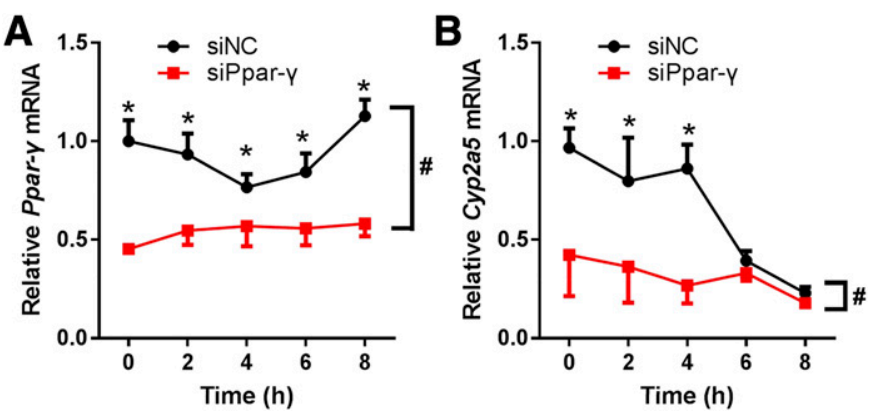

Fig. 6. Ppar- $\gamma$ knockdown weakens Cyp2a5 rhythmicity in serum-shocked Hepa$1 \mathrm{c} 1 \mathrm{c} 7$ cells. (A) The mRNA level change of Ppar- $\gamma$ in Hepa-1c1c7 cells after siPpar$\gamma$ transfection and serum shock. (B) The mRNA level change of Cyp2a5 in Hepa-1c1c7 cells after siPpar- $\gamma$ transfection and serum shock. Data shown are the mean \pm S.D. $(n=4)$. " Significant effect of siPpar- $\gamma$ treatment on gene expression $\left(P<0.05\right.$, two-way ANOVA); ${ }^{*} P<0.05$ compared with the value for the treatment group at the corresponding times (two-way ANOVA followed by Tukey's post hoc test). siNC, short interfering RNA for negative control. 
A

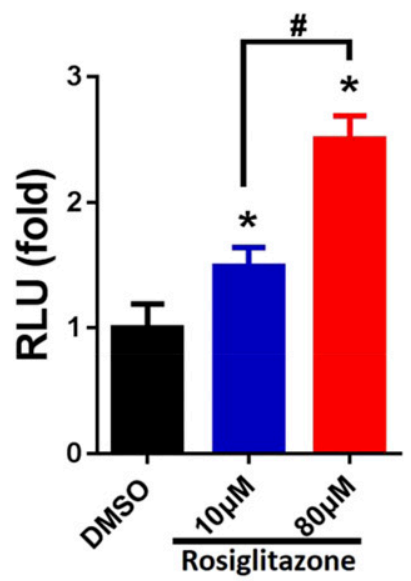

B
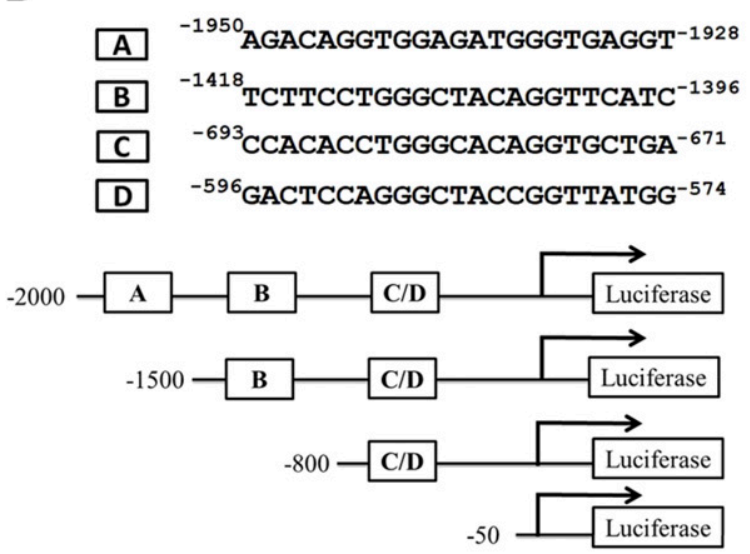

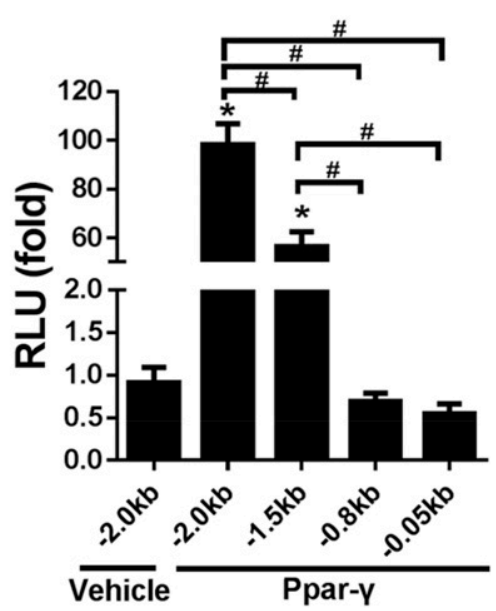

Fig. 7. Effects of Ppar- $\gamma$ activation (A) and Ppar- $\gamma$ overexpression (B) on transcriptional activity of Cyp2a5 promoter. For (A), Hepa$1 \mathrm{c} 1 \mathrm{c} 7$ cells were transfected for 24 hours and then treated with different concentrations of rosiglitazone for another 24 hours. For (B), Hepa-1c1c7 cells were cotransfected with different versions of Cyp2a5 luciferase reporter and Ppar- $\gamma$ expression plasmid for 24 hours. Data shown are the mean \pm S.D. $(n=4)$. $* P<$ 0.05 compared with the control group (one-way ANOVA followed by Tukey's post hoc test); ${ }^{\#} P<0.05$ (one-way ANOVA followed by Tukey's post hoc test). DMSO, dimethylsulfoxide; RLU, Relative Luciferase Unit.
(Fig. 8B). Taken together, our data indicated that Ppar- $\gamma$ transcriptionally activated Cyp2a5 expression via direct binding to the -1418 to -1396 -bp region (B-site).

\section{Discussion}

In this study, we showed that the expression and activity of hepatic Cyp2a5 enzyme were circadian time-dependent (Figs. 1 and 2). More importantly, our study was the first to demonstrate that the nuclear receptor Ppar- $\gamma$ was a transcriptional activator of Cyp2a5, and its rhythmic expression contributed to the circadian expression of Cyp2a5 (Figs. 3 and 7). The evidence for transcriptional regulation of Cyp2a5 by Ppar- $\gamma$ was strong. First, Ppar- $\gamma$ activation by the selective agonist rosiglitazone upregulated Cyp2a5 expression in Hepa-1c1c7 cells, whereas Ppar- $\gamma$ knockdown downregulated Cyp2a5 expression (Figs. 4 and 5). Second, use of rosiglitazone and overexpression of Ppar- $\gamma$ consistently induced the promoter activity of Cyp2a5 in luciferase reporter assays (Fig. 7). Third, based on a combination of promoter truncation, EMSA, and ChIP assays, Ppar- $\gamma$ directly bound to a PPRE (i.e., the -1418- to -1396-bp region) within Cyp2a5 promoter and activated the gene transcription (Figs. 7 and 8). Cyp2a5 contributes extensively to metabolism and detoxification of various drugs (e.g., valproic acid, disulfiram, and losigamone) and chemicals (e.g., coumarin and nicotine) (Malaiyandi et al., 2005). Identification of Ppar- $\gamma$ as a pharmacokinetic determinant of Cyp2a5 substrates assumes great importance, as the knowledge would facilitate a better understanding of varied pharmacokinetics and possibly pharmacodynamics of Cyp2a5 substrates.

We proposed that circadian regulation of Cyp2a5 by Ppar- $\gamma$ protein was attained through the rhythmic expression of this positive regulator. This was because: 1) circadian Ppar- $\gamma$ protein level was highly correlated with circadian Cyp2a5 mRNA level (Fig. 3C), and 2) knockdown of Ppar- $\gamma$ blunted the rhythmicity of Cyp2a5 expression in serum-shocked Hepa-1c1c7 cells (Fig. 6). This type of mechanism for generation of circadian gene was also noted previously for Cyp 2 b10 (Gachon et al., 2006). Circadian expression of Cyp2b10 was not directly produced by the clock output genes (i.e., the three PAR bZip transcription factors DBP, TEF [thyrotroph embryonic factor], and
A

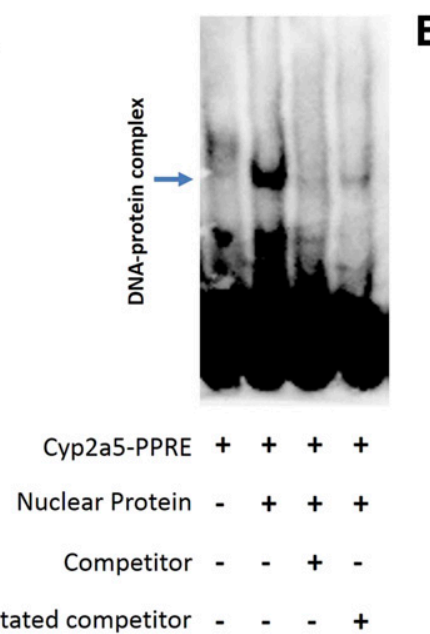

B

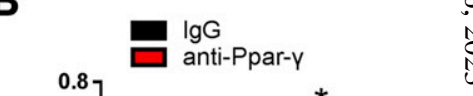

Fig. 8. Interactions of Ppar- $\gamma$ with Cyp2a5 promoter. (A) Ppar- $\gamma$ binds to Cyp2a5 promoter (B-site). EMSAs were performed with labeled Cyp2a5 promoter probe in the presence or absence of nuclear extracts from Ppar- $\gamma$-overexpressing Hepa-1c1c7 cells. (B) ChIP assays showing recruitment of Ppar- $\gamma$ protein to Cyp2a5 promoter (B-site). Data shown are the mean \pm S.D. $(n=4) .{ }^{*} P<0.05$ ( $t$ test $)$. 
HLF [hepatic leukemia factor]) but generated by the rhythmic constitutive androstane receptor, which is a transcriptional activator of Cyp2b10 (Gachon et al., 2006). The serum-shock method was used herein to investigate the effect of Ppar- $\gamma$ on cellular rhythmic expression of Cyp2a5 because a serum shock can induce circadian gene expression in the cells (whereas a regular cell culture cannot) (Edvardsson et al., 1999). This method has been successfully used to study the molecular mechanisms for circadian regulation of other P450 enzymes, including CYP2D6, CYP2E1, and CYP3A4 (Takiguchi et al., 2007; Matsunaga et al., 2008, 2012). Considering the instantaneous effect of siRNA (for Ppar- $\gamma$ silencing), the serum-shock experiments were conducted for 8 hours. Even within this relatively short period, both Ppar- $\gamma$ and Cypa 25 mRNA display obvious fluctuations (Fig. 6). The rhythmicity of Cyp2a5 mRNA was strongly dampened in Ppar- $\gamma$ knockdown cells, supporting a critical role for Ppar- $\gamma$ in circadian regulation of Cyp2a5 (Fig. 6).

Hepatic Cyp2a5 protein in mice peaked at the night-to-day transition time, consistent with a previous study (Lavery et al., 1999). This translates to circadian time dependence of metabolite generation of 7-hydroxycoumarin from coumarin (a metabolic pathway highly associated with Cyp2a5; Lake, 1999) both in vitro and in vivo (Fig. 2). Despite altered coumarin metabolism, there was no significant change in the coumarin pharmacokinetic curve (Fig. 2A). This was probably because Cyp2a5-mediated 7-hydroxylation accounts for a minor portion of total coumarin metabolism in mice (Lake, 1999), and an alteration in 7-hydroxylation cannot produce a significant change in coumarin pharmacokinetics. However, we argue for a high possibility for coumarin chronopharmacokinetics in humans because, in humans, 7-hydroxylation (mediated by the ortholog enzyme CYP2A6) is the predominant metabolism pathway for coumarin (Miles et al., 1990).

Previous studies have identified Ppar- $\alpha$ and Ppar- $\delta$ as the regulators of P450 enzymes (Barclay et al., 1999; Thomas et al., 2013; Koga et al., 2016). Therefore, combined with current findings, all three PPAR proteins have great potential to modulate the expression and activity of P450s (the principal enzymes for drug metabolism), suggesting a potential role for PPAR nuclear receptors in drug metabolism and disposition. Since PPARs are the therapeutic targets of many drugs ( $\mathrm{Li}$ et al., 2008), PPAR activation by drug substances would also modify the activities of drug-metabolizing enzymes, thereby accounting for drug efficacy modulation and drug-drug interactions.

In summary, mouse hepatic Cyp2a5 and Ppar- $\gamma$ were identified as circadian genes, and circadian Ppar- $\gamma$ protein level was strongly correlated with Cyp2a5 mRNA level. Further, Ppar- $\gamma$ activation upregulated Cyp2a5 expression in Hepa-1c1c7 cells, whereas Ppar- $\gamma$ knockdown downregulated Cyp2a5 expression. Interestingly, Ppar- $\gamma$ knockdown also blunted Cyp2a5 rhythmicity in serum-shocked Hepa-1c1c7 cells. According to a combination of promoter truncation, EMSA, and ChIP assays, Ppar- $\gamma$ directly bound to a PPRE (i.e., the -1418 - to $-1396-b p$ region) within Cyp $2 a 5$ promoter and activated the gene transcription. Taken together, Ppar- $\gamma$ was a transcriptional activator of Cyp2a5, and its rhythmic expression contributed to circadian expression of Cyp2a5.

\section{Authorship Contributions}

Participated in research design: Deng, Guo, Wu.

Conducted experiments: Deng, Guo.

Performed data analysis: Deng, Guo, Wu.

Wrote or contributed to the writing of the manuscript: Deng, Wu.

\section{References}

Balsalobre A, Damiola F, and Schibler U (1998) A serum shock induces circadian gene expression in mammalian tissue culture cells. Cell 93:929-937.
Barbier O, Villeneuve L, Bocher V, Fontaine C, Torra IP, Duhem C, Kosykh V, Fruchart JC, Guillemette C, and Staels B (2003) The UDP-glucuronosyltransferase 1A9 enzyme is a peroxisome proliferator-activated receptor alpha and gamma target gene. J Biol Chem 278 13975-13983.

Barclay TB, Peters JM, Sewer MB, Ferrari L, Gonzalez FJ, and Morgan ET (1999) Modulation of cytochrome P-450 gene expression in endotoxemic mice is tissue specific and peroxisome proliferator-activated receptor-alpha dependent. J Pharmacol Exp Ther 290:1250-1257.

Bogaards JJ, Bertrand M, Jackson P, Oudshoorn MJ, Weaver RJ, van Bladeren PJ, and Walther B (2000) Determining the best animal model for human cytochrome P450 activities: a comparison of mouse, rat, rabbit, dog, micropig, monkey and man. Xenobiotica 30:1131-1152.

Choudhary D, Jansson I, Stoilov I, Sarfarazi M, and Schenkman JB (2005) Expression patterns of mouse and human CYP orthologs (families 1-4) during development and in different adult tissues. Arch Biochem Biophys 436:50-61.

Dallmann R, Brown SA, and Gachon F (2014) Chronopharmacology: new insights and therapeutic implications. Annu Rev Pharmacol Toxicol 54:339-361.

Dallmann R, Okyar A, and Lévi F (2016) Dosing-time makes the poison: circadian regulation and pharmacotherapy. Trends Mol Med 22:430-445.

DeBruyne JP, Weaver DR, and Dallmann R (2014) The hepatic circadian clock modulates xenobiotic metabolism in mice. J Biol Rhythms 29:277-287.

Desvergne B and Wahli W (1999) Peroxisome proliferator-activated receptors: nuclear control of metabolism. Endocr Rev 20:649-688.

Edvardsson U, Bergström M, Alexandersson M, Bamberg K, Ljung B, and Dahllöf B (1999) Rosiglitazone (BRL49653), a PPARgamma-selective agonist, causes peroxisome proliferatorlike liver effects in obese mice. J Lipid Res 40:1177-1184.

Fujita K and Kamataki T (2001) Predicting the mutagenicity of tobacco-related N-nitrosamines in humans using 11 strains of Salmonella typhimurium YG7108, each coexpressing a form of human cytochrome P450 along with NADPH-cytochrome P450 reductase. Environ Mol Mutagen 38:339-346.

Gachon F, Olela FF, Schaad O, Descombes P, and Schibler U (2006) The circadian PAR-domain basic leucine zipper transcription factors DBP, TEF, and HLF modulate basal and inducible xenobiotic detoxification. Cell Metab 4:25-36.

Guengerich FP (1997) Comparisons of catalytic selectivity of cytochrome P450 subfamily enzymes from different species. Chem Biol Interact 106:161-182.

Guengerich FP (2008) Cytochrome p450 and chemical toxicology. Chem Res Toxicol 21:70-83. Hanukoglu I (1992) Steroidogenic enzymes: structure, function, and role in regulation of steroid hormone biosynthesis. J Steroid Biochem Mol Biol 43:779-804.

Honkakoski P and Negishi M (1997) The structure, function, and regulation of cytochrome P450 2A enzymes. Drug Metab Rev 29:977-996.

Hrycay EG and Bandiera SM (2009) Expression, function and regulation of mouse cytochrome P450 enzymes: comparison with human P450 enzymes. Curr Drug Metab 10:1151-1183.

Jones G, Prosser DE, and Kaufmann M (2014) Cytochrome P450-mediated metabolism of vitamin D. J Lipid Res 55:13-31.

Jones JR, Barrick C, Kim KA, Lindner J, Blondeau B, Fujimoto Y, Shiota M, Kesterson RA, Kahn BB, and Magnuson MA (2005) Deletion of PPARgamma in adipose tissues of mice protects against high fat diet-induced obesity and insulin resistance. Proc Natl Acad Sci USA 102 $6207-6212$.

Koga T, Yao PL, Goudarzi M, Murray IA, Balandaram G, Gonzalez FJ, Perdew GH, Fornace AJ, $\mathrm{Jr}$, and Peters JM (2016) Regulation of cytochrome P450 2B10 (CYP2B10) expression in liver by peroxisome proliferator-activated Receptor- $\beta / \delta$ modulation of SP1 promoter occupancy. $J$ Biol Chem 291:25255-25263.

Lake BG (1999) Coumarin metabolism, toxicity and carcinogenicity: relevance for human risk assessment. Food Chem Toxicol 37:423-453.

Lavery DJ, Lopez-Molina L, Margueron R, Fleury-Olela F, Conquet F, Schibler U, and Bonfils C (1999) Circadian expression of the steroid 15 alpha-hydroxylase (Cyp2a4) and coumarin 7-hydroxylase (Cyp2a5) genes in mouse liver is regulated by the PAR leucine zipper transcription factor DBP. Mol Cell Biol 19:6488-6499.

Lévi F, Okyar A, Dulong S, Innominato PF, and Clairambault J (2010) Circadian timing in cancer treatments. Annu Rev Pharmacol Toxicol 50:377-421.

Li Y, Qi Y, Huang TH, Yamahara J, and Roufogalis BD (2008) Pomegranate flower: a unique traditional antidiabetic medicine with dual PPAR-alpha/-gamma activator properties. Diabetes Obes Metab 10:10-17.

Liu W, Li J, Liu M, Zhang H, and Wang N (2015) PPAR- $\gamma$ promotes endothelial cell migration by inducing the expression of Sema3g. J Cell Biochem 116:514-523.

Lorbek G, Lewinska M, and Rozman D (2012) Cytochrome P450s in the synthesis of cholesterol and bile acids--from mouse models to human diseases. FEBS J 279:1516-1533.

Malaiyandi V, Sellers EM, and Tyndale RF (2005) Implications of CYP2A6 genetic variation for smoking behaviors and nicotine dependence. Clin Pharmacol Ther 77:145-158.

Matsunaga N, Ikeda M, Takiguchi T, Koyanagi S, and Ohdo S (2008) The molecular mechanism regulating 24-hour rhythm of CYP2E1 expression in the mouse liver. Hepatology 48:240-251.

Matsunaga N, Inoue M, Kusunose N, Kakimoto K, Hamamura K, Hanada Y, Toi A, Yoshiyama Y, Sato F, Fujimoto K, et al. (2012) Time-dependent interaction between differentiated embryo chondrocyte- 2 and CCAAT/enhancer-binding protein $\alpha$ underlies the circadian expression of CYP2D6 in serum-shocked HepG2 cells. Mol Pharmacol 81:739-747.

Miles JS, McLaren AW, Forrester LM, Glancey MJ, Lang MA, and Wolf CR (1990) Identification of the human liver cytochrome P-450 responsible for coumarin 7-hydroxylase activity. Biochem $J$ 267:365-371

Murakami Y, Higashi Y, Matsunaga N, Koyanagi S, and Ohdo S (2008) Circadian clock-controlled intestinal expression of the multidrug-resistance gene mdr1a in mice. Gastroenterology 135 1636-1644.e3

Muruganandan S and Sinal CJ (2008) Mice as clinically relevant models for the study of cytochrome P450-dependent metabolism. Clin Pharmacol Ther 83:818-828.

Nelson DR, Zeldin DC, Hoffman SM, Maltais LJ, Wain HM, and Nebert DW (2004) Comparison of cytochrome P450 (CYP) genes from the mouse and human genomes, including nomenclature recommendations for genes, pseudogenes and alternative-splice variants. Pharmacogenetics 14: $1-18$

Partch CL, Green CB, and Takahashi JS (2014) Molecular architecture of the mammalian circadian clock. Trends Cell Biol 24:90-99.

Patten CJ, Smith TJ, Friesen MJ, Tynes RE, Yang CS, and Murphy SE (1997) Evidence for cytochrome $\mathrm{P} 4502 \mathrm{~A} 6$ and $3 \mathrm{~A} 4$ as major catalysts for $\mathrm{N}^{\prime}$-nitrosonornicotine alpha-hydroxylation by human liver microsomes. Carcinogenesis 18:1623-1630. 
Rakhshandehroo M, Knoch B, Müller M, and Kersten S (2010) Peroxisome proliferator-activated receptor alpha target genes. PPAR Res 2010:2010.

Reppert SM and Weaver DR (2002) Coordination of circadian timing in mammals. Nature 418 935-941.

Shimada T, Yamazaki H, Mimura M, Inui Y, and Guengerich FP (1994) Interindividual variations in human liver cytochrome P-450 enzymes involved in the oxidation of drugs, carcinogens and toxic chemicals: studies with liver microsomes of 30 Japanese and 30 Caucasians. J Pharmacol Exp Ther 270:414-423.

Takiguchi T, Tomita M, Matsunaga N, Nakagawa H, Koyanagi S, and Ohdo S (2007) Molecular basis for rhythmic expression of CYP3A4 in serum-shocked HepG2 cells. Pharmacogenet Genomics 17:1047-1056.

Thomas M, Burk O, Klumpp B, Kandel BA, Damm G, Weiss TS, Klein K, Schwab M, and Zanger UM (2013) Direct transcriptional regulation of human hepatic cytochrome P450 3A4 (CYP3A4) by peroxisome proliferator-activated receptor alpha (PPAR $\alpha)$. Mol Pharmacol 83:709-718.
Yi M, Shin JG, and Lee SJ (2017) Expression of CYP4V2 in human THP1 macrophages and its transcriptional regulation by peroxisome proliferator-activated receptor gamma. Toxicol Appl Pharmacol 330:100-106.

Zhang T, Zhao M, Lu D, Wang S, Yu F, Guo L, Wen S, and Wu B (2018) REV-ERB $\alpha$ regulates CYP7A1 through repression of liver receptor Homolog-1. Drug Metab Dispos 46:248-258.

Zhao M, Zhang T, Yu F, Guo L, and Wu B (2018) E4bp4 regulates carboxylesterase 2 enzymes through repression of the nuclear receptor Rev-erb $\alpha$ in mice. Biochem Pharmacol 152:293-301.

Address correspondence to: Dr. Baojian Wu, College of Pharmacy, Jinan University, 601 Huangpu Avenue West, Guangzhou 510632, China. E-mail: bj.wu@ hotmail.com 